University of Konstanz

Department of Economics

\title{
Forecasting Euro-Area Macroeconomic Variables using a Factor Model Approach for Backdating
}

\author{
Ralf Brüggemann and Jing Zeng
}

Working Paper Series

2012-15 


\title{
Forecasting Euro-Area Macroeconomic Variables Using a Factor Model Approach for Backdating*
}

\author{
Ralf Brüggemann $^{a} \quad$ Jing Zeng ${ }^{b}$
}

August 2, 2012

\begin{abstract}
We suggest to use a factor model based backdating procedure to construct historical Euro-area macroeconomic time series data for the pre-Euro period. We argue that this is a useful alternative to standard contemporaneous aggregation methods. The paper investigates for a number of Euro-area variables whether forecasts based on the factorbackdated data are more precise than those obtained with standard area-wide data. A recursive pseudo-out-of-sample forecasting experiment using quarterly data is conducted. Our results suggest that some key variables (e.g. real GDP, inflation and long-term interest rate) can indeed be forecasted more precisely with the factor-backdated data. Keywords: forecasting, factor model, backdating, European monetary union, constructing EMU data
\end{abstract}

JEL classification: C22, C53, C43, C82

\footnotetext{
${ }^{*}$ We thank participants in the DIW Berlin Macroeconometric Workshop 2009, the research seminar of the Center of Quantitative Methods and Survey Research, Konstanz, the EEA 2010 Glasgow, the CFE 2010 London, and the ESEM 2011 Oslo for helpful comments and suggestions.

${ }^{a}$ Corresponding author: Ralf Brüggemann, Universität Konstanz, Department of Economics, Box 129, 78457 Konstanz, Germany, email: ralf.brueggemann@uni-konstanz.de

${ }^{b}$ Universität Konstanz, Department of Economics, Box 129, 78457 Konstanz, Germany, email: jing.zeng@uni-konstanz.de
} 


\section{Introduction}

With the creation of European Monetary Union (EMU), the focus of macroeconomic analysis has shifted towards the analysis of the Euro area as a whole. Econometric models for area-wide variables have been used for forecasting and structural analysis. As actual EMU time series data are only available from 1999 onwards, synthetic time series data for the pre-EMU period are in use. Often the construction of historical (pre-EMU) Euro area data is based on contemporaneous aggregation of time series from the EMU member countries. Different aggregation methods have been suggested in the literature and Marcellino (2004) points out a number of drawbacks inherent in these methods. The choice of a particular aggregation method is a very important practical issue that impacts any following econometric analysis. For instance, Bosker (2006) illustrates that estimated cointegration parameters change substantially with the choice of the aggregation method. Given these drawbacks of standard methods, it is worth to consider the merits of alternatives to aggregation. In this paper, we therefore consider a factor model based alternative to the standard method of contemporaneous aggregation and analyze the usefulness of this approach in forecasting Euro area aggregates.

One of the standard aggregation methods suggested in the literature has been discussed by Fagan, Henry \& Mestre (2001, 2005). Their approach has been used to create a database of historical euro-area time series data for estimating the Area Wide Model (AWM) in use at the European Central Bank (ECB) 1 The AWM data is based on cross-country aggregation of loglevel variables with fixed weights (referred to as FHM weights). The FHM weights are obtained as shares of GDP at constant 1995 prices. Anderson, Dungey, Osborn \& Vahid (2011) point out that the use of fixed weights will tend to undervalue the importance of the countries, which hold a leading role in the European markets and suggest extending the FHM weights with a sliding factor which measures the relative distance from economic integration to EMU. Using fixed weights may also be problematic because it does not

\footnotetext{
${ }^{1}$ Updates of this database is available from the Euro Area Business Cycle Network (EABCN) at http://www.eabcn.org/
} 
take changes in exchange rates between member countries into consideration. Therefore, Beyer, Doornik \& Hendry (2001) suggest to aggregate growth rates of the variables with time-varying weights based on previous period's real GDP share (henceforth BDH weights) and find that in their method the aggregates of the individual deflators correspond to the deflator of the aggregate. Recently, Beyer \& Juselius (2009) show that results based on $\mathrm{BDH}$ weights are sensitive to the choice of base year and therefore suggest to use weights based on previous period's nominal GDP. None of the proposed methods seems optimal in all respects.

Alternatives to standard aggregation have also been considered in the literature. For instance, Brüggemann \& Lütkepohl (2006) and Brüggemann, Lütkepohl \& Marcellino (2008) argue that the use of synthetically constructed, aggregated data is inappropriate especially in the presence of structural changes induced by adjustment processes required in some countries prior to EMU in order to satisfy the Maastricht criteria. They suggest a representative country approach which combines German data until 1998 with actual Euro-area data after 1999. They find that at least for some variables like interest rates and prices using German data rather than aggregated EMU data for the pre-EMU period is preferable when forecasts of EMU aggregates are of interest.

This paper proposes to use another alternative method for constructing historical Euro-area data. We extend the idea put forward in Angelini, Henry \& Marcellino (2006) and Angelini \& Marcellino (2011), where a factor based approach is used to construct time series of macroeconomic variables for unified Germany prior to 1991. In the factor model approach, a small number of factors are extracted from a large set of time series from individual EMU member countries using the Stock \& Watson (2002a) principal component based estimators. The estimated relation between the factor time series and the actual Euro-area time series of interest is used to construct time series data for the pre-EMU period. This method is referred to as factor-backdating. Advantages of this method include its ability to use more time series information than standard aggregation methods and its ability to handle situations with missing time series data in some of the cross-sectional units (countries). Against the background of future EMU enlargement and 
the doubtful quality of historical data in some of the future member countries, the factor-backdating procedure may be an attractive and useful alternative to standard aggregation methods.

We analyze the usefulness of this approach in forecasting a number of macroeconomic Euro-area variables by conducting a forecast comparison. We compare the accuracy of forecasts based on models that use different historical Euro-area time series. In particular, we compare forecasts based on pre-EMU data from the AWM database in use at the ECB to forecasts based on data obtained from the factor-backdating procedure. Our paper is related to work by Marcellino (2004), which also includes a forecasting comparison for EMU macroeconomic time series based aggregated data constructed by Fagan, Henry \& Mestre (2005). However, the focus in Marcellino (2004) is on the forecasting performance of different forecasting methods, not on different data. Our work is also related to the study by Brüggemann et al. (2008) who investigate whether German data before the Euro period contain the same or more information for forecasting than the aggregated data by comparing linear and nonlinear forecasting methods. They find that at least for nominal and monetary variables German data results in superior forecasts.

Our study uses a number of linear and nonlinear forecasting methods and models. In particular, we include variants of linear autoregressive models as well as non-linear smooth transition regression models. These forecasting models have also been used in e.g. Stock \& Watson (1999), Marcellino (2004) and Brüggemann et al. (2008). Variables included in our comparison are real GDP, the GDP deflator, a consumer price index, short- and long-term interest rates as well as the exchange rate.

The structure of the remaining paper is as follows. In Section 2, the factorbackdating approach is presented. The forecasting methods are discussed in Section 3, before the data are described in Section 4. Section 5 discusses the results from our forecasting comparison and Section 6 concludes.

\section{Factor-based backdating}

As an alternative to standard contemporaneous aggregation methods, we suggest to use a factor-based approach to backdate historical data for the 
Euro-area. In this factor-backdating procedure, a small number of common factors is extracted from a possibly large set of time series data coming from individual Euro-area countries. Using the period where both, the information on the actual aggregated Euro-area time series (for the period after 1999) and the extracted factor time series are available, we estimate the relation between the unobserved factors and the area-wide aggregate. This information is in turn used to backdate historical Euro-area data. This approach has been suggested by Angelini et al. (2006) and used in the context of backdating German data by Angelini \& Marcellino (2011). A detailed description of the factor backdating procedure in our context is given in the following.

Starting point is a factor model representation discussed by Stock \& Watson $(2002 \mathrm{a}, 2002 \mathrm{~b})$. In their approach the $N$-dimensional stationary time series $X_{t}$ is driven by a small number of $K$ unobserved common factors $F_{t}$ and an idiosyncratic component $e_{t}$, i.e. the vector of time series may be written as

$$
X_{t}=\Lambda F_{t}+e_{t}, \quad t=1, \ldots, T,
$$

where $X_{t}$ is a $N \times 1$ vector, $\Lambda$ is a $N \times K$ matrix of factor loadings, $F_{t}$ is the $K \times 1$ vector of common factors and $e_{t}$ is an $N \times 1$ vector of idiosyncratic components. Prior to the backdating procedure, the common factors have to be extracted from the time series data. Estimation of the factors is done using a classical static principle components on $\tilde{X}_{t}$, which is obtained by standardizing $X_{t}$ to have mean zero and unit variance. This procedure gives a $K$-dimensional time series of common factors, denoted as $\left\{\hat{F}_{t}\right\}_{t=1}^{T}$. It can be shown that under mild regularity conditions the principal components of $\tilde{X}_{t}$ are consistent estimators of the true unobservable factors (see e.g. Stock \& Watson (2002a) for details). In applications, the variables in $X_{t}$ are transformed to stationarity if necessary. The choice of the number of factors may be based on suitable criteria (see e.g. Bai \& Ng (2002)).

In our application, we split the entire sample period (from 1970Q1 to 2010Q4) into a estimation period (from 1970Q1 to $T_{1}$ ) and a forecasting evaluation period (from $T_{1}+1$ to 2010Q4). In the following we set $T_{1}$ to 2002Q4 and thus the forecasting evaluation starts in 2003Q1. The vector $X_{t}$ consists of a number of time series coming from the individual Euro-area 
member countries and we extract the factor time series using data over the period from 1970Q1 to 2002Q4. In the second step, we relate the factor time series to the area-wide macroeconomic series of interest using a period, where observations on both are available. To be more precise, we regress the (stationarity transform) of the Euro-area-wide series of interest, denoted as $y_{t}^{E M U}$, on the estimated factor time series $\hat{F}_{t}$ over the period from $1999 Q 1$ to $2002 Q 4$, i.e. we use

$$
y_{t}^{E M U}=\beta_{0}+\beta_{1} \hat{F}_{1 t}+\ldots+\beta_{K} \hat{F}_{K t}+\varepsilon_{t}, \quad t=1999 Q 1, \ldots, 2002 Q 4 .
$$

and estimate the parameters $\beta_{0}, \ldots, \beta_{K}$ by OLS. In the third step of our procedure, we use the estimated parameters $\hat{\beta}_{0}, \ldots, \hat{\beta}_{K}$ to backdate the (historical) area-wide time series for the periods before 1999 by:

$$
\hat{y}_{t}^{E M U}=\hat{\beta}_{0}+\hat{\beta}_{1} \hat{F}_{1 t}+\ldots+\hat{\beta}_{K} \hat{F}_{K t}, \quad t=1970 Q 1, \ldots, 1998 Q 4 .
$$

This factor model approach has several advantages: While it uses the information from all member countries like standard aggregation methods do, it avoids the difficulty to choose the appropriate aggregation weights. In the factor-backdating method, the weights are obtained in a data driven way. Moreover, standard aggregation methods typically only use the country information on the one variable that is aggregated. In the factor based approach, $X_{t}$ can in principle include many other variables as well. For instance, when constructing Euro-area data for the overall consumer price index, the vector $X_{t}$ used in the factor backdating may include the time series of consumer price subindexes or from other price indices as well. Therefore, the information content of an area-wide time series obtained by the described factor method may be greater than in a time series obtained by standard aggregation methods. The factor-based method is also suitable to handle missing time series observations in some cross-sectional units, a situation that occurs often when constructing Euro-area aggregates. Even if there are some missing observations in some of the cross-sections, the common factors can still be extracted by using the expectation-maximization (EM) algorithm (see e.g. the discussion in Appendix A of Stock \& Watson (2002a) and in Angelini et al. (2006)). Against the background of future EMU enlargement and the doubtful quality of historical data in some of the future member countries, 
the factor backdating procedure may be an attractive and useful alternative to standard aggregation methods.

The usefulness of the suggested approach in forecasting is investigated in the remaining part of the paper.

\section{Forecasting Methods and Evaluation}

\subsection{Forecasting Methods}

The forecasting methods used in this work are similar to those discussed by Stock \& Watson (1999), Marcellino (2004) and Brüggemann et al. (2008). Thus, only a brief description of the different methods are given in the following.

In our forecasting exercise we are interested in forecasting the EMU aggregate of some variable of interest $h$ periods ahead. We denote this variable as $y_{t}^{E M U}$. Depending on the integration properties of this variable, the forecasting model is either specified for the level $y_{t}^{E M U}$ or for the first difference $\Delta y_{t}^{E M U}=y_{t}^{E M U}-y_{t-1}^{E M U}$. To make the forecast errors comparable across both cases, we specify forecasting models for the variable $y_{t+h}^{h}$, where $y_{t+h}^{h}=y_{t+h}^{E M U}$, when the variable is stationary and $y_{t+h}^{h}=y_{t+h}^{E M U}-y_{t}^{E M U}$, when the variable is integrated of order one $(I(1))$. $h$ denotes the forecasting horizon. All considered forecasting methods can be written as

$$
y_{t+h}^{h}=f\left(Z_{t} ; \theta_{h t}\right)+\varepsilon_{t+h},
$$

where $Z_{t}$ is the vector of explanatory variables, $\theta_{h t}$ is a vector of possibly time-varying parameters and $\varepsilon_{t}$ is an error term. The $h$-step ahead forecast is given by replacing the unknown parameter vector $\theta_{h t}$ by an estimate and hence,

$$
\hat{y}_{t+h}^{h}=f\left(Z_{t} ; \hat{\theta}_{h t}\right),
$$

and the $h$-step forecast error is

$$
e_{t+h}=y_{t+h}^{h}-\hat{y}_{t+h}^{h}=y_{t+h}-\hat{y}_{t+h} \text {. }
$$


We use $h=1, h=2$ and $h=4$ as forecasting horizons. In the case of multi-step predictions, we use the so-called ' $h$-step ahead projection', which is also known as the 'direct forecast' approach (see e.g. Clements \& Hendry (1996)). In other words, different forecasting models are fitted for different forecasting horizons. In comparison to the 'iterated multi-step forecasts', the direct forecasting method is advantageous in the context of nonlinear models as simulation from nonlinear models is avoided.

We use linear autoregressive models as well as non-linear smooth transition regression models. The model variants are briefly described in the following:

Autoregressions (AR). This simple linear forecasting method has the form

$$
y_{t+h}^{h}=\mu_{t}+\beta^{\prime} Z_{t}+\varepsilon_{t+h} .
$$

If $y_{t}^{E M U}$ is treated as a stationary variable, then $Z_{t}=\left(y_{t}, \ldots, y_{t-p+1}\right)^{\prime}$, otherwise $Z_{t}=\left(\Delta y_{t}, \ldots, \Delta y_{t-p+1}\right)^{\prime}$, where the superscript $E M U$ has been dropped to simplify the notation. In the deterministic component $\mu_{t}$ a constant or a constant and a time trend can be included. Three variants of lag lengths are considered: a fixed number of lags $p=4$; lag length selected by AIC $(0 \leq p \leq 4)$; lag length selected by $\mathrm{BIC}(0 \leq p \leq 4)$. Since the variable $y_{t}^{E M U}$ can be treated as stationary, or as $I(1)$, or a unit root pre-test may be used, there are 18 model variants in this class. The different variants are denoted as A1-A18 and listed in Panel A of Table 1

Logistic smooth transition autoregression (LSTAR). This nonlinear forecasting method is of the form

$$
y_{t+h}^{h}=\alpha^{\prime} Z_{t}+d_{t} \beta^{\prime} Z_{t}+\varepsilon_{t+h} .
$$

As in the autoregressive models, $Z_{t}=\left(1, y_{t}, \ldots, y_{t-p+1}\right)^{\prime}$ if $y_{t}$ is treated stationary or $Z_{t}=\left(1, \Delta y_{t}, \ldots, \Delta y_{t-p+1}\right)^{\prime}$ if $y_{t}$ is integrated. The term $d_{t}$ is a logistic function $d_{t}=1 /\left[1+\exp \left(\gamma_{0}+\gamma_{1} \zeta_{t}\right)\right]$. The value of the so-called smoothing parameters $\gamma_{1}$ determines the shape of parameter change over time. For $\gamma_{1}=0$, the model becomes linear, while for large values of $\gamma_{1}$ the model tends to a self-exciting threshold model, see e.g. Granger \& Teräsvirta (1993) and 
Teräsvirta (1998) for details. $\zeta_{t}$ is the transition variable and in the considered variants may depend on current and past $y_{t}$. For models specified in levels, the following five alternatives are used for $\zeta_{t}: \zeta_{t}=y_{t} ; \zeta_{t}=y_{t-1}$; $\zeta_{t}=y_{t-3} ; \zeta_{t}=y_{t}-y_{t-2} ; \zeta_{t}=y_{t}-y_{t-4}$. The choice of the transition variable follows Marcellino (2004). For models specified in first differences, the following five alternatives are used for $\zeta_{t}: \zeta_{t}=\Delta y_{t} ; \zeta_{t}=\Delta y_{t-1} ; \zeta_{t}=\Delta y_{t-3}$; $\zeta_{t}=y_{t}-y_{t-2} ; \zeta_{t}=y_{t}-y_{t-4}$. The lag length $p$ of the model is fixed 2 in some models, while in some other variants we use AIC and BIC to select from a choice of models with $p=1,2,4$ and all possible $\zeta_{t}$ mentioned above. This gives a total of 12 different LSTAR model variants, denoted as L1-L12. The models used are listed in Panel B of Table 1.

\subsection{Forecasting Comparison}

In this work we do not focus on the comparison of forecasting methods, but investigate whether the AWM data or factor-backdated data is preferable for making forecasts. For this purpose, we conduct a recursive pseudo-out-ofsample forecasting experiment and look at forecasting precision at horizons $h=1,2$ and 4 . In our experiment, the initial estimation period ranges from 1970Q1 to 2002Q4 and forecasting evaluation is done over the period 2003Q1 to 2010Q4. Thus, the initial estimation period has $T_{1}=132$ observations and the forecasting period covers $T_{2}-T_{1}=32$ quarters. To mimic the behavior of a forecaster, the unit root pre-tests, model selection and estimation are repeated once a new observation is added to the estimation period.

To compare the forecasting performance, the mean squared forecast error (MSFE) is used as loss function. For forecast horizon $h$, model $m$ and variable $n$ with type of data $j$ it can be defined as:

$$
\operatorname{MSFE}_{n, m, j}^{h}=\frac{1}{T_{2}-T_{1}-h+1} \sum_{t=T_{1}}^{T_{2}-h}\left(e_{t+h, n, m, j}\right)^{2},
$$

where the forecast error is $e_{t+h}=y_{t+h}^{h}-\hat{y}_{t+h}^{h}$. To simplify the comparison, each MSFE obtained from the factor-backdating approach, denoted as, $\mathrm{MSFE}_{n, m, F}^{h}$ will be expressed relative to the MSFE obtained from models based on AWM data, denoted as $\mathrm{MSFE}_{n, m, A W M}^{h}$. Thus, if the relative MSFE 
is less than one, the forecasts based on factor backdated data are more precise than forecasts based on AWM data. For all forecasting models, the results were adjusted. That is, if a forecasted change exceeds in absolute value any change previously observed for that series, it will be replaced by a no-change forecast $2^{2}$

\section{Data}

Our forecasting comparison includes six Euro-area macroeconomic variables on a quarterly frequency: real GDP (YER), the GDP deflator (YED), the consumer price index (CPI), the exchange rate against the US-Dollar (EER) and short- and long-term interest rates (STN and LTN). The mnemonics correspond to those in the AWM database.

The first set of area-wide time series corresponds to data obtained from the AWM database maintained at the Euro Area Business Cycle Network. ${ }^{3}$ As mentioned before, the AWM data is based on cross-country aggregation of log-level variables with fixed weights. The aggregation methods is the one used in Fagan, Henry \& Mestre (2001) and Fagan et al. (2005). This AWM data is now in widespread use, e.g. within the ECB for estimating econometric models. It should be noted, however, that the AWM database is not an official ECB database. Quarterly data for the period from 1970Q1 to 2010Q4 4 are used in the following and the corresponding time series plots are given in Figure 1 .

As an alternative we consider sets of time series obtained from variants of the backdating procedure described in Section 2, For this procedure the individual member countries' time series data are taken from the OECD quarterly national accounts database and are available for a period from 1970Q1 to 2007Q45 For the interest rate variables STN and LTN missing obser-

\footnotetext{
${ }^{2}$ This adjustment was only needed in some of the nonlinear forecasting methods.

${ }^{3}$ http://www.eabcn.org

${ }^{4}$ The $11^{\text {th }}$ update of the AWM database in 2011 is considered. This update extends the AWM data up to 2010Q4.

${ }^{5}$ Twelve euro-zone countries are considered: Austria, Belgium, Finland, France, Germany, Greece, Italy, Ireland, Luxembourg, Netherlands, Portugal, Spain. The data are obtained via Thomson Datastream.
} 
vations are present such that the expectation-maximization (EM) algorithm is applied to construct a balanced panel ${ }^{6}$ For illustrative purposes, Figure 1 depicts for each considered variable time series of the three largest Euroarea member states Germany, France, and Italy. For some variables, like e.g. price measures and interest rates, the time series plots reflect quite different developments in the three countries. In the forecasting exercise below, we consider the log-transform of real GDP, the GDP deflator, the consumer price index and the exchange rate, while short- and long-term interest rates are not transformed. Time series on the variables for all twelve considered countries are characterized by trends and their is evidence that the series can be characterized as $I(1)$ processes $7^{7}$ Therefore, the first difference of the variables enters the vector $X_{t}$, which after standardization is used to estimate the common factors.

In this study the first backdating variant extracts factors from a set of country time series data that consists only of the variable corresponding to the aggregate of interest. For instance, when backdating area-wide real GDP the factors are extracted from a set $X_{t}$ that only includes real GDP growth from the member countries and no other variables are taken into account. We refer to this backdating variant as F1 backdating (or F1 data) in the following. Since in the factor approach $X_{t}$ may also include many other variables as well, in the second variant we extract the factors used in backdating from all considered variables using data from the twelve EMU member countries. For example, for the factor backdating of the variable real GDP, the factors are extracted by considering all the six variables YER, YED, CPI, EER, STN and LTN from 12 countries 8 This backdating variant is referred to as F2 backdating (F2 data) in the following.

\footnotetext{
${ }^{6}$ For the short-term interest rate STN, 56 missing values can be observed for Ireland from 1970Q1 to 1983Q4, 28 missing values for Spain from 1970Q1 to 1976Q4 and 4 missing for Italy values for the year 1979. For the long-term interest rate LTN, there are 108 missing observations for Greece from 1970Q1 to 1996Q4, 96 missing observations for Luxembourg from 1970Q1 to 1993Q4, and 4 missing observations for Ireland in 1979.

${ }^{7} \mathrm{~A}$ formal unit root analysis has been conducted and the results are available on request.

${ }^{8}$ For the variable YED only 11 countries data are available. For Greece GDP deflator data is not available in standard databases.
} 
The number of factors (principle components) $L$ used in backdating has to be determined in practical applications. When using the information criteria proposed by Bai \& Ng (2002), we find that all three criteria suggest the maximum number of factors in the F1 backdating variant. Given the relatively low number of time series in $X_{t}$, this does not seem be a useful choice. Therefore, we decided to explore the cumulative percentage of the variance in $X_{t}$ that is explained by the principle components. Table 2 reports for each variable the respective percentage of explained variance for the first 10 factors when data until 2002Q4 is used to extract the factor time series. Results for the first variant of factor extraction (F1) are given in columns (1) to (6). Clearly, there is some variation in the percentage of explained variance over the different variables. For instance, the first factor extracted from the 12 EMU time series on real GDP growth (YER) accounts for not more than $28.1 \%$ of the total variation, which might reflect the fairly heterogeneous developments in the real economies of the Euro-area member states. In contrast, for the exchange rate variable EER $83.6 \%$ of the variation can be explained by its first factor, which may be due to the German dominance within the European Monetary System (EMS). We decided to use two factors in F1 backdating for each of the variables and this choice ensures that for each variable at least $35 \%$ of the total variance in the respective data set is explained.

When all $(N=12 \times 6)$ variables are considered in the $\mathrm{F} 2$ variant, the first two factors explain $16.7 \%$ and $12.1 \%$ of the total variation, respectively (cf. column (7) in Table 2). In line with the choice in the F1 variant, we decided to use two factors in F2 backdating 9

The factor backdating procedure described in Section 2 is applied for the six mentioned variables, where factor extraction is over the period 1970Q12002Q4 and the corresponding backdating is for the period 1970Q1-1998Q4. The forecasting covers the period from 2003Q1 to 2010Q4. As we treat all variables as $I(1)$, the backdating is done on the first differences of the

\footnotetext{
${ }^{9}$ One exception is the exchange rate variable EER, for which three factors are used to avoid negative values in the backdated data. In the $\mathrm{F} 2$ variant, we also used the criteria by Bai \& Ng (2002). They typically suggested a slightly larger number of factors that used here.
} 
respective variables. From the backdated changes (and growth rates) we compute the respective level of the time series. This approach gives two sets of six factor-backdated Euro-area time series and plots of these series are given in Figure 2 .

Comparing the area-wide series from the AWM database with those obtained by the factor backdating procedure (see Figure 2p shows that for the pre-Euro period both methods lead to time series that have similar trending behavior. Nevertheless, the medium and short-term fluctuations are typically quite different, which in turn may have an impact on the forecasting performance. From 1999Q1 onwards, time series from both approaches are identical because both use actual Euro-area data.

\section{Results}

The results from our forecasting comparison for all six variables are presented in Figures 3 to 8. For each variable and forecasting model variant (see Table 1), we report the MSFE of the model based on factor-backdated data relative to the MSFE of the corresponding model based on AWM data. Results for forecasting horizons $h=1,2$ and 4 are reported in the upper, middle and bottom panel of the corresponding figure, respectively. The black bars refer to factor-backdating based on factors extracted from EMU country data on the variable to be predicted (F1 variant), while bars with diagonal crosshatches refer to factor-backdating based on all six variables (F2 variant). The AWM database with data up to 2010Q4 is used. The forecasting evaluation period starts in 2003Q1.

Figure 3 shows the results for real GDP (YER). For $h=1$, the overall forecasting performance of models using factor-backdated data tends to be better than for models based on AWM data. Compared to using AWM data, both factor-backdating variants lead to sizable gains in forecasting precision in all considered linear model specifications (Models 1 to 18). Interestingly, in these linear models using the backdating variant F2, i.e. using more variables in factor extraction, leads to consistently lower MSFEs than using the F1 backdating. Obviously, taking more information into account helps to increase the forecasting precision at short horizons. We also note that using the 
factor-backdated data leads to substantial gains in forecasting precision in most of the nonlinear forecasting models. For $h=2$, using factor-backdated data leads to smaller MSFEs (compared to using AWM data) in a number of linear and non-linear forecasting models. For the linear models we find, however, also some relative MSFEs around one models indicating that both data variants perform equally well in predicting the real GDP. Gains tend to be larger in non-linear models when using the backdating variant F1, possibly pointing the some non-linearities in the underlying DGP. For horizon $h=4$, we again find that the use of factor-backdated data variant F1 is beneficial with some sizable gains in forecasting precision in most of the forecasting models.

Figure 4 shows the results for the GDP deflator (YED). For $h=1$ and $h=2$, the forecasting performance of linear models using factor-backdated data from variant F1 is slightly better than of models using the AWM data in most specifications. Also note that for this variable using more information in the F2 backdating variants does lead to slightly larger MSFEs compared to the F1 variant. Moreover, the use of factor-backdated data does not seem to be beneficial if the forecasts are based on non-linear models. In fact, using the F2 backdating variant leads to inferior forecasts in almost all nonlinear forecasting models. At forecasting horizon $h=4$, the different data variants almost all lead to comparable forecasting accuracy. Exceptions are the models 4, 10, and 16, which are AR models in first differences of the log price deflator with a linear trend. This model implies that the quarterly GDP deflator inflation rate has a linear deterministic trend and these AR models perform quite well in predicting the GDP deflator using factor-backdated data. Most likely these specifications pick up the downward trend in the GDP deflator inflation rate during the EMU run-up period and may therefore lead to large gains in forecasting precision.

The results for the consumer price index (CPI, see Figure 5) indicate that using the factor-backdating variant F2 leads to notably higher forecasting accuracy than using AWM data in all considered linear autoregressive specifications and at all forecasting horizons. In contrast, sizable gains for the F1 backdating variant are only observed at $h=1$. For larger prediction horizons these gains tend to disappear. For the nonlinear forecasting 
models the results are less clear-cut. While some gains of using backdated data are visible for some model specifications, we also find some nonlinear models where using AWM data leads to more precise forecasts. This suggests that careful model selection is particularly important in nonlinear models. Overall, using the factor-backdated data based on all variables (variant F2) in combination with linear models for the CPI seems to be beneficial with substantial gains in MSFEs at both, short and long prediction horizons.

For the exchange rate variable (EER, see Figure 6) using the factorbackdated data is generally not beneficial in linear models when the focus is on the short forecasting horizons $h=1$ and $h=2$. Most of the relative MSFEs are around one, indicating that both data variants perform equally well in predicting the exchange rate. Interestingly, some of the nonlinear LSTAR variants with backdated time series outperform their AWM counterparts. These results are more clear-cut at higher forecasting horizons. For $h=4$, using factor-backdated data is beneficial with substantial reductions in MSFE in some of the forecasting models, especially in the nonlinear ones. In forecasting the exchange rate, the two backdating variants lead generally to very comparable forecasts and there is not much to choose here.

For the long term interest rate (LTN) (see Figure 7) we find for all forecasting horizons that using factor-backdated data based on only one variable (variant F1) gives more precise forecasts than using their AWM data counterparts in almost all considered forecasting models. Exceptions are only visible for some of the nonlinear prediction models. We also observe that using more information in the backdating variant F2 leads to larger MSFEs compared to the F1 backdating. This may indicate that the key information is already contained in the long-term interest rate series of the EMU countries. If the information on the other variables is not relevant for the long-term interest rate, then adding these additional variables to the larger data set used in factor extraction may add some noise and the key information useful for interest rates can no longer be extracted precisely enough from the larger data set (see Boivin \& Ng (2006) for a related discussion).

Figure 8 shows the results for the short-term interest rate (STN). For all prediction horizons most of the MSFEs are around one, so the backdated data and the AWM data have similar forecasting performance and nothing is 
gained by using the backdated data. Some nonlinear variants with backdated data lead to rather large relative MSFEs at $h=1$. This points to the fact that the corresponding model is not well specified and highlights the need of careful model specification when using nonlinear models in forecasting.

Table 3 shows for each of the considered variables and for each forecasting horizon the three best performing model/data variants together with the corresponding relative MSFEs. Note that we have now used the same benchmark model for all models in order facilitate the comparison across different specifications. The benchmark is an $\mathrm{AR}(4)$ with a constant, specified in levels for the variable using AWM data. This is an alternative way to summarize the results of our forecasting comparison and can be employed to judge the usefulness of the factor-backdating approach. The results in Table 3 indicate, for instance, that at all horizons, the three best forecasting models for real GDP and the long term interest rate are models that use factor-backdated pre-euro data. In line with the results discussed above, we find that the best forecasting models for the long-term interest rate are those based on the backdating variant $\mathrm{F} 1$. In addition, we find for all variables (with the exception of the GDP deflator) that the best performing models at forecasting horizon $h=1$ is based on factor-backdating data. Thus, using factor-backdated data for the pre-euro period is a useful strategy when forecasts with short horizons are of interest.

We have conducted a number of robustness checks in our analysis. First, for both, F1 and F2 backdating, we tried different number of factors in backdating. Of course, this leads to some changes in the forecasting results but the overall results are similar to those reported above and are therefore not discussed here. Moreover, we have also used different splits into estimation and forecasting evaluation periods. We note some smaller changes but again the overall results are similar to the ones discussed above. Thus, for the sake of brevity we have not included them here. All additional results are available on request.

Overall, our results indicate that for some key variables like the real GDP, the CPI inflation rate and the long-term interest rate using factor-backdated data for the pre-euro period is a useful strategy when forecasts are of interest. 


\section{Conclusion}

In this paper we have suggested to use a factor model based backdating procedure to construct historical Euro-area macroeconomic time series data for the pre-Euro period. We argue that this is a useful alternative to standard contemporaneous aggregation methods as it may be used in situations where time series data from some cross-sectional units is missing or not available in the desired quality. Against the background of future EMU enlargement and the doubtful quality of historical data in some of the future member countries, the factor-backdating procedure may be an attractive and useful alternative to standard aggregation methods.

We have conducted a recursive pseudo-out-of-sample forecasting experiment to investigate for a number of Euro-area variables whether forecasts based on the factor-backdated data are more precise than those obtained with standard area-wide (AWM) data. Our results suggests that some key variables like real GDP, long-term interest rates and CPI inflation can indeed be forecasted more precisely with the factor backdated data.

Overall, our results indicate that for some important variables the factorbackdating procedure is a valuable method to construct time series data for the Euro-area. 


\section{References}

Anderson, H., Dungey, M., Osborn, D. R. \& Vahid, F. (2011). Financial integration and the construction of historical financial data for the euro area, Economic Modelling 28(4): 1498-1509.

Angelini, E., Henry, J. \& Marcellino, M. (2006). Interpolation and backdating with a large information set, Journal of Economic Dynamics and Control 30: 2693-2724.

Angelini, E. \& Marcellino, M. (2011). Econometric analyses with backdated data - unified Germany and the euro area, Economic Modelling 28(3): 1405-1414.

Bai, J. \& Ng, S. (2002). Determining the number of factors in approximate factor models, Econometrica 71(1): 135-172.

Beyer, A., Doornik, J. A. \& Hendry, D. F. (2001). Constructing historical euro-zone data, The Economic Journal 111: F102-F121.

Beyer, A. \& Juselius, K. (2009). Does it matter how to measure aggregates? the case of monetary transmission mechanisms in the euro area, The Methodology and Practice of Econometrics: A Festschrift in Honour of David F. Hendry. Castle, J. L. and Shepard, N. (eds), Oxford University Press.

Boivin, J. \& Ng, S. (2006). Are more data always better for factor analysis?, Journal of Econometrics 132(1): 169-194.

Bosker, E. M. (2006). On the aggregation of eurozone data, Economics Letters 90: 260-265.

Brüggemann, R., Lütkepohl, H. \& Marcellino, M. (2008). Forecasting euro-area variables with German pre-EMU data, Journal of Forecasting 27: 465-481.

Brüggemann, R. \& Lütkepohl, H. (2006). A small monetary system for the euro area based on German data, Journal of Applied Econometrics 21(6): $683-702$. 
Clements, P. M. \& Hendry, D. F. (1996). Multi-step estimation for forecasting, Oxford Bulletin of Economics and Statistics 58: 657-684.

Fagan, G., Henry, J. \& Mestre, R. (2001). An area-wide model for the Euro area, Working Papers 42, European Central Bank.

Fagan, G., Henry, J. \& Mestre, R. (2005). An area-wide model for the euro area, Economic Modelling 22: 39-59.

Granger, C. W. J. \& Teräsvirta, T. (1993). Modelling non-linear economic relationships, Oxford: Oxford University Press .

Marcellino, M. (2004). Forecasting EMU macroeconomic variables, International Journal of Forecasting 20: 359-372.

Stock, J. H. \& Watson, M. W. (1999). A comparison of linear and non-linear univariate models for forecasting macroeconomic time series, Cointegration, causality, and forecasting: A festschrift in honor of Clive $W$. J. Granger. Engle, R. and White, R. (eds), Oxford University Press.

Stock, J. H. \& Watson, M. W. (2002a). Macroeconomic forecasting using diffusion indexes, Journal of Business and Economic Statistics 20: 147162.

Stock, J. H. \& Watson, M. W. (2002b). Forecasting using principal components from a large number of predictors, Journal of the American Statistical Association 97: 1167-1179.

Teräsvirta, T. (1998). Modelling economic relationships with smooth transition regressions, Handbook of Applied Economic Statistics. Ullah, A. and Giles, D. E. A. (eds), New York: Marcel Dekker. 
Table 1: Forecasting models

\begin{tabular}{l|l}
\hline \hline \multicolumn{2}{|c}{ A. Linear models: Autoregressive models (18 variants) } \\
\hline A1 & AR(4) in levels with constant \\
A2 & AR(4) in levels with linear trend \\
A3 & AR(4) in first differences with constant \\
A4 & AR(4) in first differences with linear trend \\
A5 & AR(4) with constant, pretested for unit root \\
A6 & AR(4) with linear trend, pretested for unit root \\
A7 & AR in levels with constant, AIC for lag length \\
A8 & AR in levels with linear trend, AIC for lag length \\
A9 & AR in first differences with constant, AIC for lag length \\
A10 & AR in first differences with linear trend, AIC for lag length \\
A11 & AR with constant, pretested for unit root, AIC for lag length \\
A12 & AR with linear trend, pretested for unit root, AIC for lag length \\
A13 & AR in levels with constant, BIC for lag length \\
A14 & AR in levels with linear trend, BIC for lag length \\
A15 & AR in first differences with constant, BIC for lag length \\
A16 & AR in first differences with linear trend, BIC for lag length \\
A17 & AR with constant, pretested for unit root, BIC for lag length \\
A18 & AR with linear trend, pretested for unit root, BIC for lag length \\
\hline \hline B. Nonlinear models: Logistic smooth transition autoregressions (12 variants) \\
\hline L1 & LSTAR(2) in levels, transition var. yt \\
L2 & LSTAR(2) in first differences, transition var. $y_{t}$ \\
L3 & LSTAR(2), pretested for unit root, transition var. $y_{t}$ \\
L4 & LSTAR(2) in levels, transition var. $y_{t}-y_{t-2}$ \\
L5 & LSTAR(2) in first differences, transition var. $y_{t}-y_{t-2}$ \\
L6 & LSTAR(2), pretested for unit root, transition var. $y_{t}-y_{t-2}$ \\
L7 & LSTAR in levels, AIC on transition var. and lag length \\
L8 & LSTAR in first differences, AIC on transition var. and lag length \\
L9 & LSTAR, pretested for unit root, AIC on transition var. and lag length \\
L10 & LSTAR in levels, BIC on transition var. and lag length \\
L11 & LSTAR in first differences, BIC on transition var. and lag length \\
L12 & LSTAR, pretested for unit root, BIC on transition var. and lag length \\
\hline \hline
\end{tabular}


Table 2: Percentage of explained variance for the first 10 factors

\begin{tabular}{|c|c|c|c|c|c|c|c|}
\hline \multirow[b]{3}{*}{ Factor } & \multicolumn{6}{|c|}{ factor-extraction F1 } & factor extraction F2 \\
\hline & (1) & (2) & (3) & (4) & $(5)$ & (6) & (7) \\
\hline & YER & YED & CPI & EER & LTN & STN & ALL \\
\hline 1 & 0.281 & 0.233 & 0.398 & 0.836 & 0.453 & 0.340 & 0.167 \\
\hline 2 & 0.098 & 0.182 & 0.183 & 0.077 & 0.103 & 0.124 & 0.121 \\
\hline 3 & 0.095 & 0.113 & 0.091 & 0.025 & 0.092 & 0.098 & 0.096 \\
\hline 4 & 0.091 & 0.098 & 0.077 & 0.018 & 0.064 & 0.084 & 0.058 \\
\hline 5 & 0.080 & 0.087 & 0.054 & 0.013 & 0.059 & 0.079 & 0.040 \\
\hline 6 & 0.072 & 0.069 & 0.053 & 0.011 & 0.055 & 0.067 & 0.031 \\
\hline 7 & 0.068 & 0.062 & 0.040 & 0.008 & 0.045 & 0.056 & 0.030 \\
\hline 8 & 0.053 & 0.049 & 0.028 & 0.006 & 0.043 & 0.053 & 0.026 \\
\hline 9 & 0.051 & 0.046 & 0.024 & 0.005 & 0.034 & 0.042 & 0.024 \\
\hline 10 & 0.040 & 0.036 & 0.020 & 0.002 & 0.023 & 0.029 & 0.023 \\
\hline
\end{tabular}

Note: Entries show the percentage of explained variance in the data sets due to the first 10 principle components. Column (1) to (6) show results for factors extracted only from EMU country data on the variable mentioned in the respective column (F1 variant). Column (7) shows results for data set that includes EMU country data on all six variables mentioned in columns (1) to (6) (F2 variant). YER is real GDP, YED is the GDP deflator, CPI is the consumer price index, EER is the exchange rate against the US-Dollar, and LTN and STN denote long- and short-term interest rates. Sample period for factor extraction: 1970Q1-2002Q4. 
Table 3: Best three performing forecasting models/data variants

\begin{tabular}{|c|c|c|c|c|c|c|c|c|c|}
\hline & \multicolumn{3}{|c|}{$h=1$} & \multicolumn{3}{|c|}{$h=2$} & \multicolumn{3}{|c|}{$h=4$} \\
\hline Variable & Model & Data & MSFE & Model & Data & MSFE & Model & Data & MSFE \\
\hline \multirow{3}{*}{ YER } & L4 & F1 & 0.536 & $\mathrm{~L}^{a}{ }^{a}$ & F1 & 0.664 & A14 & F1 & $\begin{array}{l}0.870 \\
\end{array}$ \\
\hline & L10 & F1 & 0.654 & $\mathrm{~L}_{1} 1^{b}$ & $\mathrm{~F} 1$ & 0.711 & $\mathrm{~A} 1$ & $\mathrm{~F} 2$ & 0.881 \\
\hline & $\mathrm{A} 7$ & $\mathrm{~F} 2$ & 0.804 & $\mathrm{~L} 8^{c}$ & F1 & 0.784 & $\mathrm{~A} 8$ & F1 & 0.885 \\
\hline \multirow{3}{*}{ YED } & $\mathrm{L} 7^{d}$ & F1 & 0.994 & $\mathrm{~A} 1^{e}$ & F1 & 0.988 & $\mathrm{~L} 7^{f}$ & AWM & 0.983 \\
\hline & L7 & AWM & 0.999 & $\mathrm{~A} 4$ & $\mathrm{~F} 2$ & 0.999 & $\mathrm{~A} 1^{g}$ & AWM & 0.999 \\
\hline & A1 & AWM & 1.000 & $\mathrm{~A} 1^{h}$ & AWM & 1.000 & $\mathrm{~L} 7$ & F1 & 1.000 \\
\hline \multirow{3}{*}{ CPI } & $\mathrm{L}^{i}{ }^{i}$ & F2 & 0.553 & L10 & $\mathrm{F} 2$ & 0.672 & L10 & F2 & 0.525 \\
\hline & $\mathrm{A} 4^{j}$ & $\mathrm{~F} 2$ & 0.584 & L1 & AWM & 0.690 & L1 & $\mathrm{F} 2$ & 0.544 \\
\hline & $\mathrm{A} 3^{k}$ & $\mathrm{~F} 2$ & 0.589 & L1 & $\mathrm{F} 2$ & 0.692 & L10 & AWM & 0.560 \\
\hline \multirow{3}{*}{ EER } & $\mathrm{L} 7^{l}$ & $\mathrm{~F} 2$ & 0.878 & $\mathrm{~L} 1^{m}$ & F1 & 0.687 & $\mathrm{~L} 7^{n}$ & F1 & 0.612 \\
\hline & $\mathrm{L} 1^{o}$ & F1 & 0.921 & $\mathrm{~L} 10^{p}$ & $\mathrm{~F} 2$ & 0.750 & $\mathrm{~L} 1^{q}$ & F1 & 0.664 \\
\hline & $\mathrm{L} 5$ & $\mathrm{~F} 1$ & 0.938 & $\mathrm{~L} 4^{r}$ & $\mathrm{~F} 2$ & 0.775 & L10 & F1 & 0.715 \\
\hline \multirow{3}{*}{ LTN } & $\mathrm{A} 2^{s}$ & $\bar{F} 1$ & 0.801 & $\overline{\mathrm{A} 2}$ & $\overline{F 1}$ & 0.712 & $\mathrm{~A} 8^{t}$ & F1 & 0.638 \\
\hline & $\mathrm{A} 3^{u}$ & F1 & 0.844 & $\mathrm{~A} 8^{v}$ & $\mathrm{~F} 1$ & 0.748 & $\mathrm{~A} 2$ & F1 & 0.639 \\
\hline & L7 & F1 & 0.845 & $\mathrm{~A} 3^{w}$ & F1 & 0.814 & $\mathrm{~A} 3^{x}$ & F1 & 0.768 \\
\hline \multirow{3}{*}{ STN } & L4 & F2 & 0.842 & $\mathrm{~A} 2$ & F1 & 0.760 & $\mathrm{~A} 8^{y}$ & F1 & 0.622 \\
\hline & A2 & F1 & 0.849 & $\mathrm{~A} 8^{z}$ & F1 & 0.771 & A2 & F1 & 0.624 \\
\hline & A 8 & F1 & 0.851 & $\mathrm{~A} 8^{a a}$ & AWM & 0.789 & $\mathrm{~A} 8^{b b}$ & AWM & 0.666 \\
\hline
\end{tabular}

Note: AWM denotes aggregated area-wide model data, F1 denotes factor-backdated data based on factors extracted only from EMU country data on the variable mentioned in the respective row (F1 variant) and F2 denotes factor-backdated data based on factors from using EMU country data on all variables mentioned in table. Entries in column 'MSFE' are MSFEs relative to benchmark model. The benchmark is an AR(4) with constant based on AWM data. Model names correspond to those from Table1. YER is real GDP, YED is the GDP deflator, CPI is the consumer price index, EER is the exchange rate against the US-Dollar, and LTN and STN denote long- and short-term interest rates. Sample period: 1970Q1-2010Q4. Forecast evaluation period: 2003Q1-2010Q4.

${ }^{a}$ L6 leads to same MSFE. ${ }^{b}$ L12 leads to same MSFE. ${ }^{c}$ L9 leads to same MSFE. ${ }^{d}$ L10 leads to same MSFE. ${ }^{e} \mathrm{~A} 7$ leads to same MSFE. ${ }^{f} \mathrm{~L} 10$ leads to same MSFE. ${ }^{g} \mathrm{~A} 7$ and A13 lead to same MSFE. ${ }^{h} \mathrm{~A} 7$ and A13 lead to same MSFE. ${ }^{i} \mathrm{~L} 9$ leads to same MSFE. ${ }^{j} \mathrm{~A} 10$ leads to same MSFE. ${ }^{k} \mathrm{~A} 5$, A6, A9, A11, A15, A17 and A18 lead to same MSFE. ${ }^{l}$ L9 leads to same MSFE. ${ }^{m}$ L3 leads to same MSFE. ${ }^{n}$ L9 leads to same MSFE. ${ }^{o}$ L3 leads to same MSFE. ${ }^{p}$ L12 leads to same MSFE. ${ }^{r}$ L6 leads to same MSFE. ${ }^{s}$ A8 leads to same MSFE. ${ }^{t}$ A14 leads to same MSFE. ${ }^{u}$ A5, A6, A9, A11 and A12 lead to same MSFE. ${ }^{v}$ A14 leads to same MSFE. ${ }^{w}$ A5, A6, A10, A11 and A12 lead to same MSFE. ${ }^{x}$ A5, A6, A9, A11 and A12 lead to same MSFE. ${ }^{y}$ A14 leads to same MSFE. ${ }^{z}$ A14 leads to same MSFE. ${ }^{a a} \mathrm{~A} 14$ leads to same MSFE. ${ }^{b b} \mathrm{~A} 14$ leads to same MSFE. 

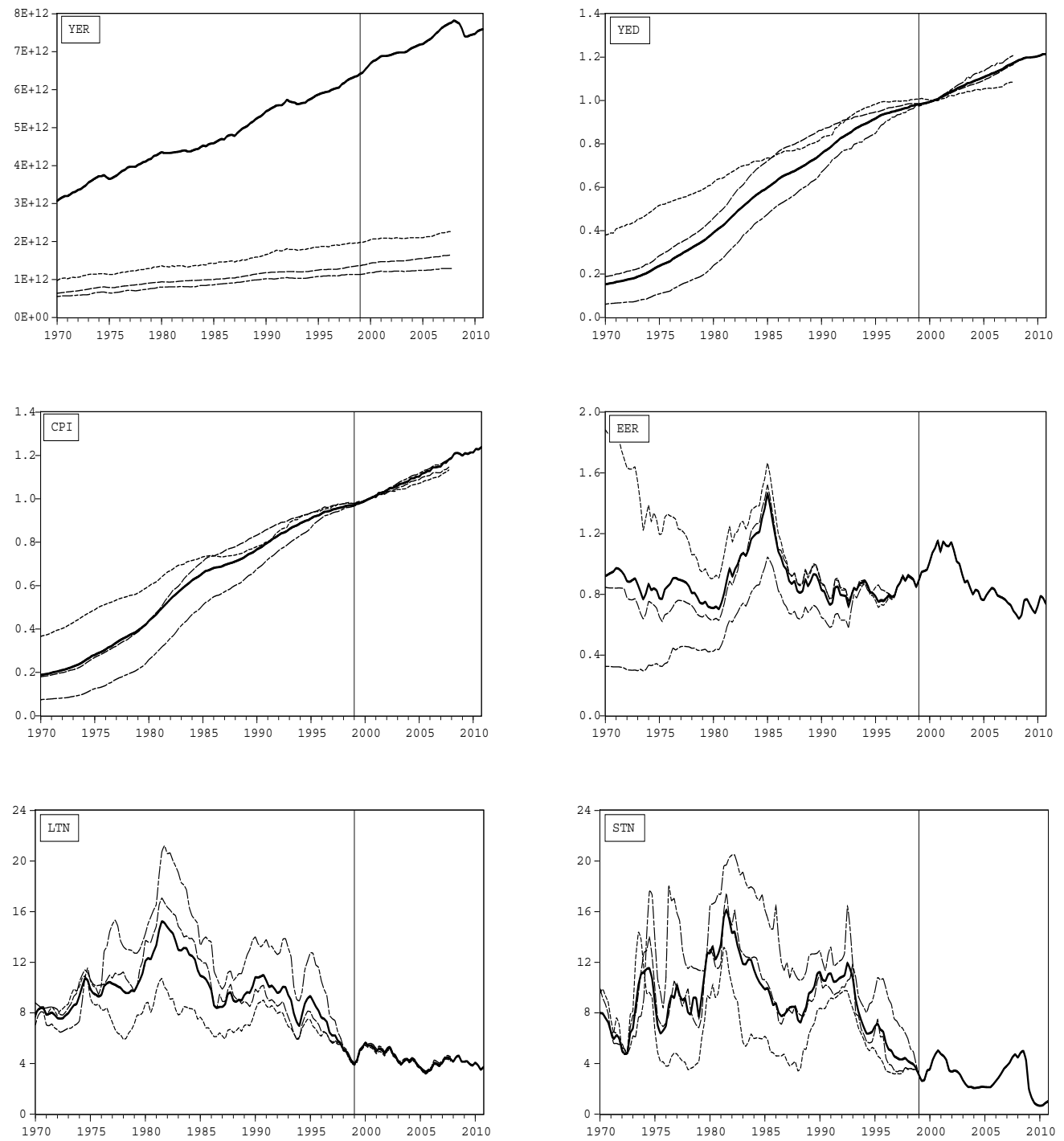

Figure 1: AWM Euro-area time series (solid lines) for real GDP (YER), the GDP deflator (YED), the consumer price index (CPI), the exchange rate (EER) and short- and long-term interest rates (STN and LTN) from 1970Q1 to 2010Q4. The dotted lines show time series plots of the corresponding variables for the three largest EMU member countries Germany, France and Italy from 1970Q1 to 2007Q4. 

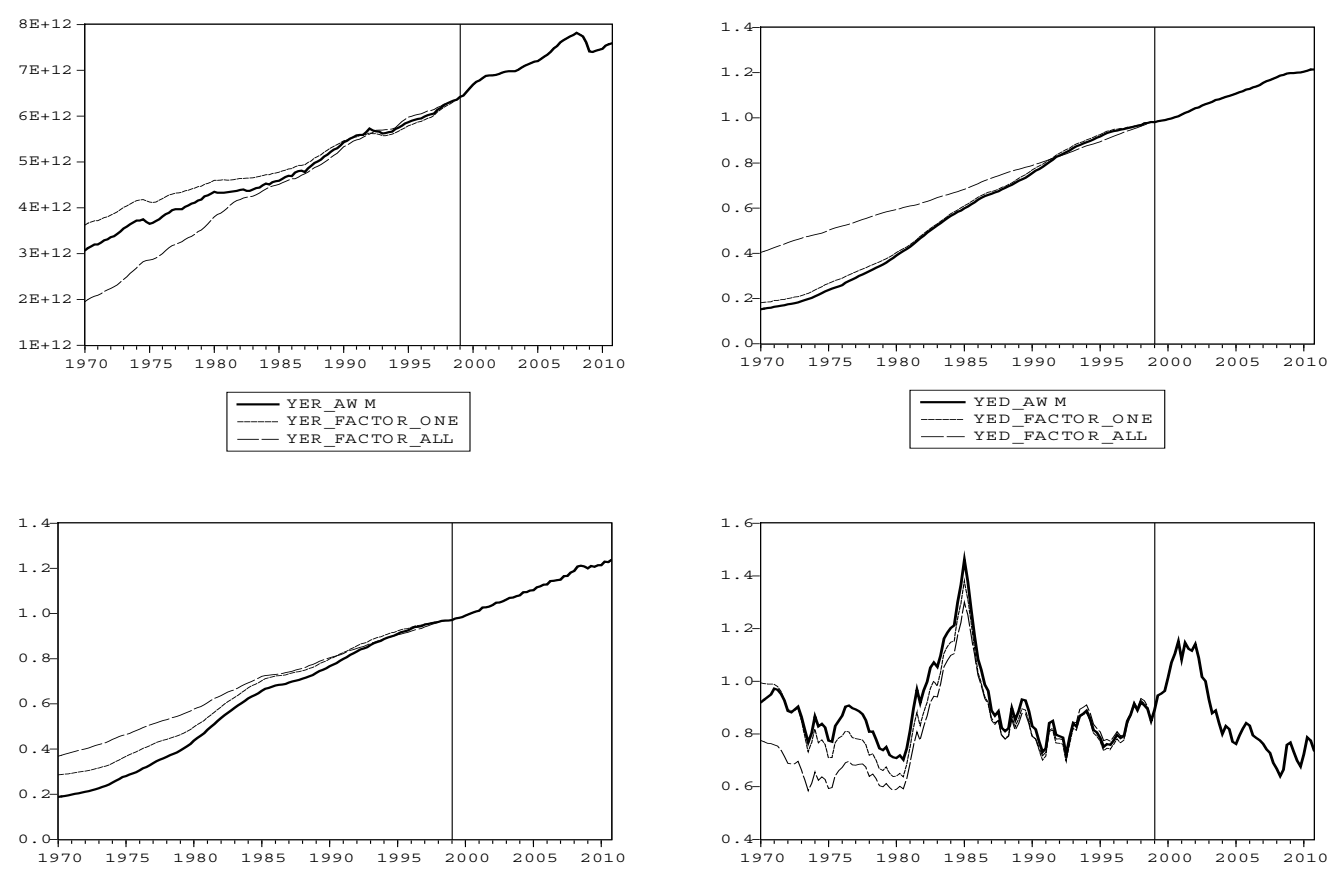

-1 CPIAWM
-- CPIFACTOR_ONE
- CPIFACTOR_ALL


Figure 2: Euro-area time series for real GDP (YER), the GDP deflator (YED), the consumer price index (CPI), the exchange rate (EER) and shortand long-term interest rates (STN and LTN). Area-wide model series (solid lines), factor-backdated time series F1 using factors extracted from only one variable (short dashed line), and factor-backdated time series F2 using factors from all variables (long dashed lines). 

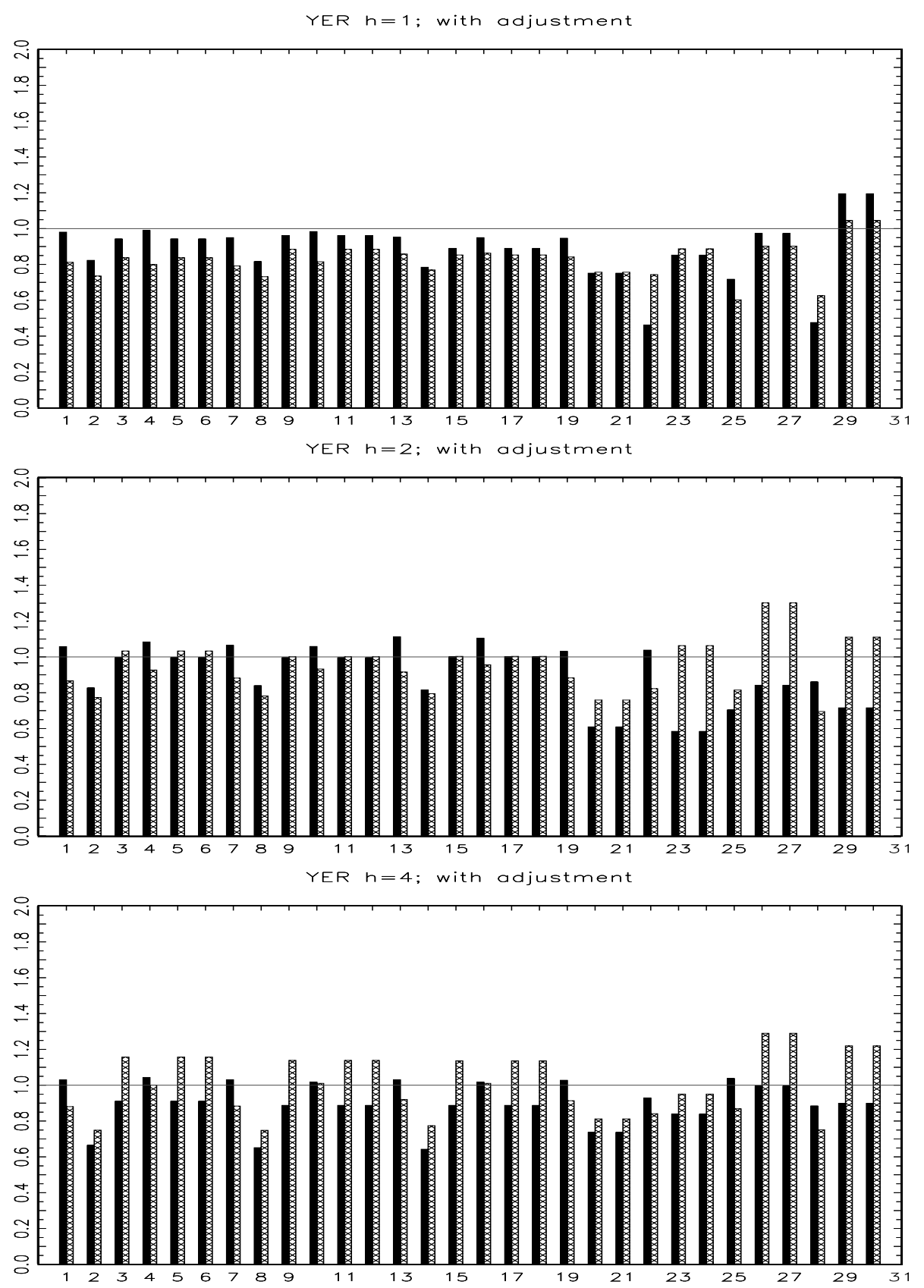

Figure 3: Results from forecasting comparison for real GDP (YER). MSFEs of models using factor-backdated data relative to corresponding model with AWM data. The bars correspond to results from the 18 linear and 12 nonlinear forecasting model variants ordered as in Table 1. Black bars refer to factor-backdating based on only one variable (variant F1), while bars with diagonal cross-hatches refer to factor-backdating based on all variables (variant F2). Sample period: 1970Q1-2010Q4. Forecast evaluation period: 2003Q12010 Q4. 



Figure 4: Results from forecasting comparison for GDP deflator (YED). MSFEs of models using factor-backdated data relative to corresponding model with AWM data. The bars correspond to results from the 18 linear and 12 nonlinear forecasting model variants ordered as in Table 1. Black bars refer to factor-backdating based on only one variable (variant F1), while bars with diagonal cross-hatches refer to factor-backdating based on all variables (variant F2). Sample period: 1970Q1-2010Q4. Forecast evaluation period: 2003Q1-2010Q4. 

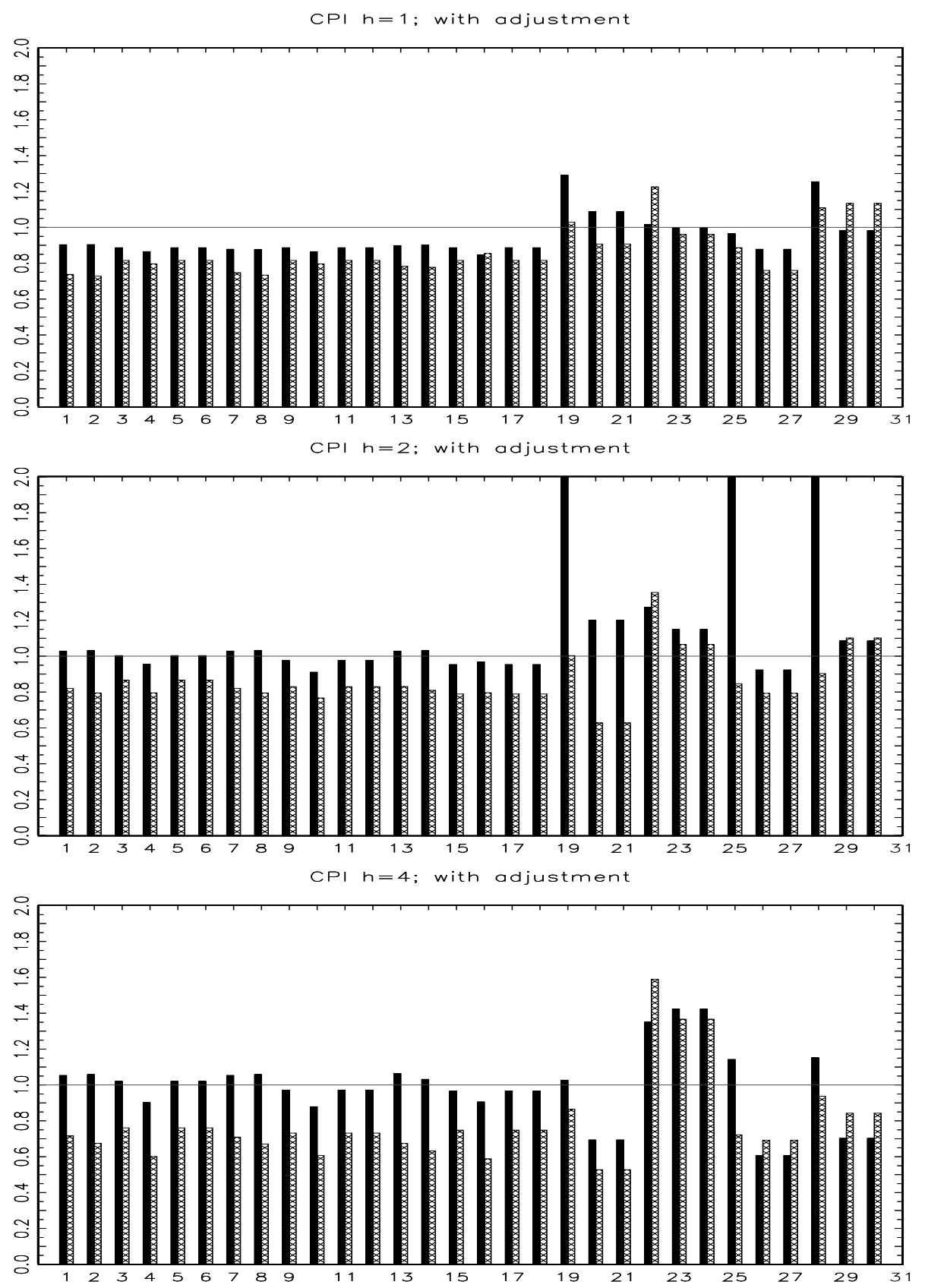

Figure 5: Results from forecasting comparison for the consumer price index (CPI). MSFEs of models using factor-backdated data relative to corresponding model with AWM data. The bars correspond to results from the 18 linear and 12 nonlinear forecasting model variants ordered as in Table1. Black bars refer to factor-backdating based on only one variable (variant F1), while bars with diagonal cross-hatches refer to factor-backdating based on all variables (variant F2). Sample period: 1970Q1-2010Q4. Forecast evaluation period: 2003Q1-2010Q4. 

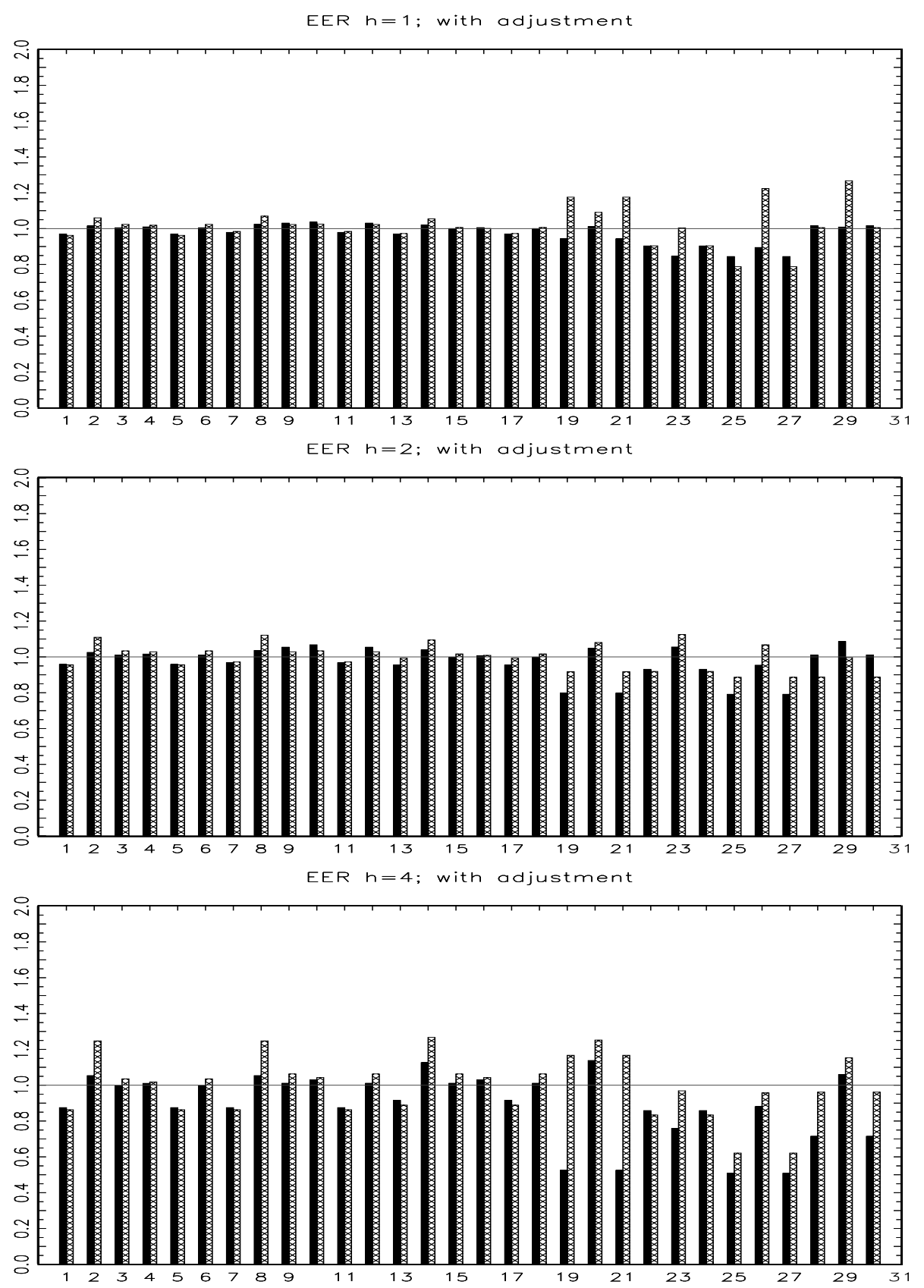

Figure 6: Results from forecasting comparison for the exchange rate (EER). MSFEs of models using factor-backdated data relative to corresponding model with AWM data. The bars correspond to results from the 18 linear and 12 nonlinear forecasting model variants ordered as in Table 1. Black bars refer to factor-backdating based on only one variable (variant F1), while bars with diagonal cross-hatches refer to factor-backdating based on all variables (variant F2). Sample period: 1970Q1-2010Q4. Forecast evaluation period: 2003Q1-2010Q4. 

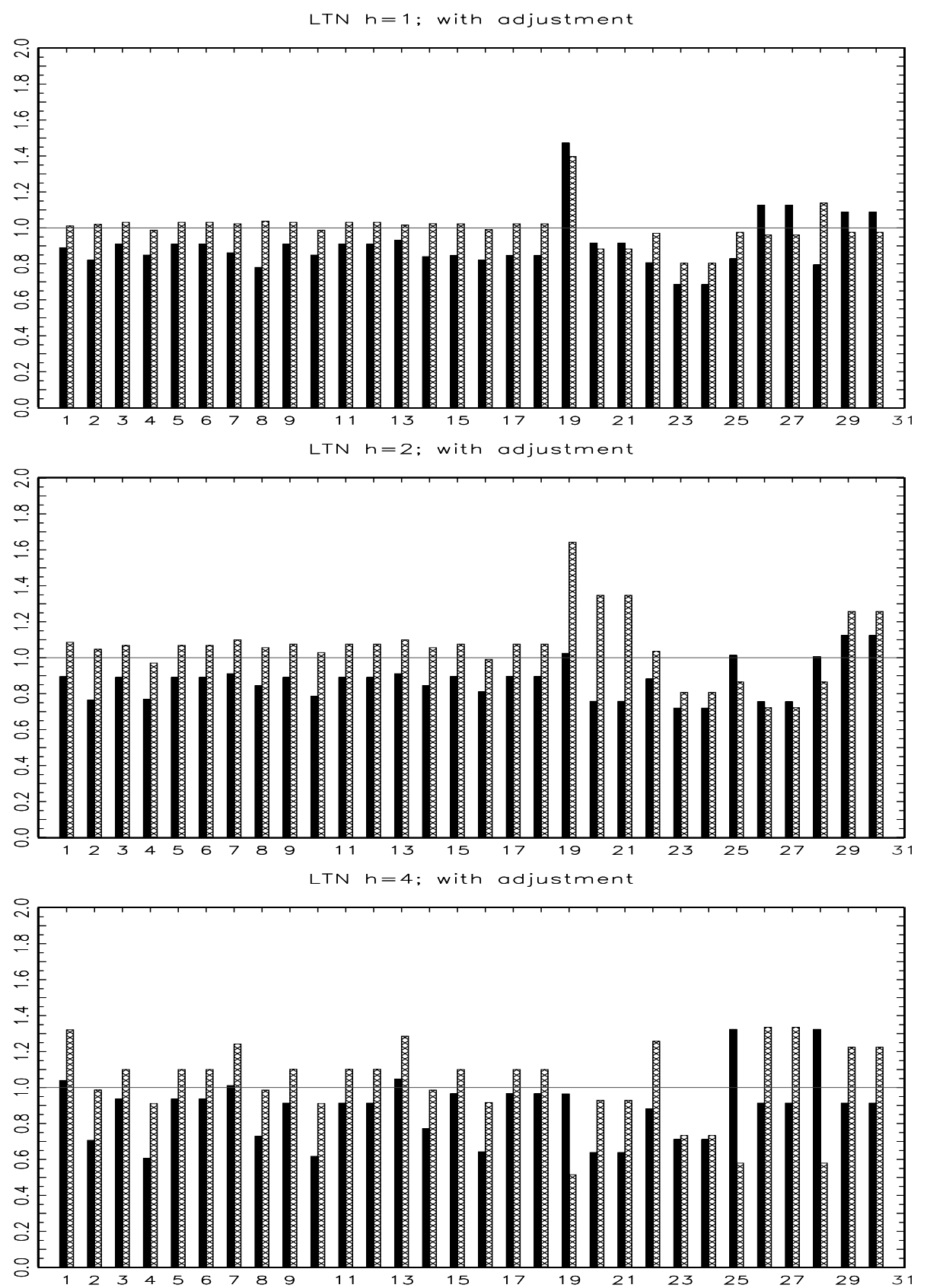

Figure 7: Results from forecasting comparison for long-term interest rate (LTN). MSFEs of models using factor-backdated data relative to corresponding model with AWM data. The bars correspond to results from the 18 linear and 12 nonlinear forecasting model variants ordered as in Table1. Black bars refer to factor-backdating based on only one variable (variant F1), while bars with diagonal cross-hatches refer to factor-backdating based on all variables (variant F2). Sample period: 1970Q1-2010Q4. Forecast evaluation period: 2003Q1-2010Q4. 

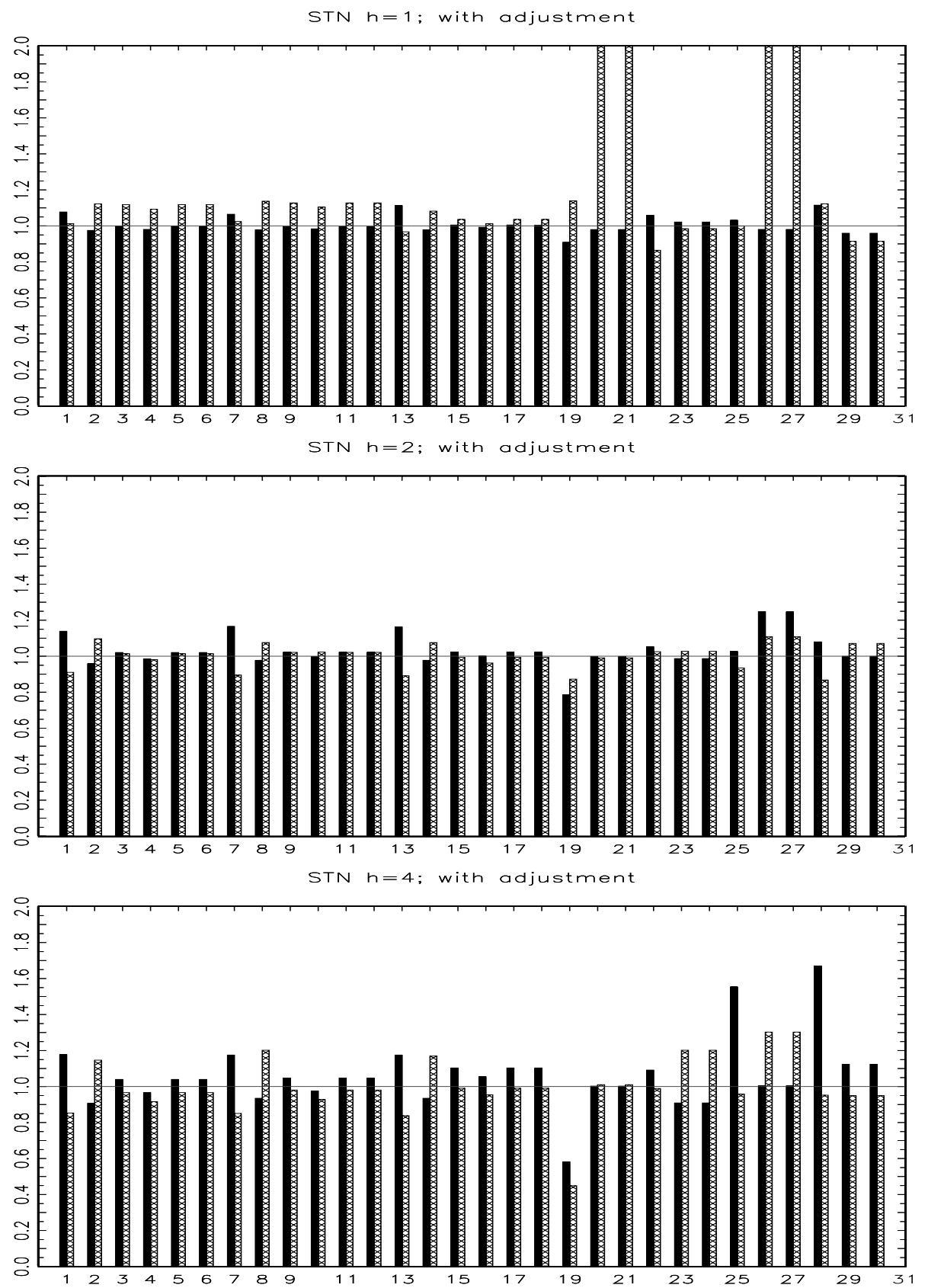

Figure 8: Results from forecasting comparison for short-term interest rate (STN). MSFEs of models using factor-backdated data relative to corresponding model with AWM data. The bars correspond to results from the 18 linear and 12 nonlinear forecasting model variants ordered as in Table1. Black bars refer to factor-backdating based on only one variable (variant F1), while bars with diagonal cross-hatches refer to factor-backdating based on all variables (variant F2). Sample period: 1970Q1-2010Q4. Forecast evaluation period: 2003Q1-2010Q4. 